\title{
Hölzl, Richard, Umkämpfte Wälder : die Geschichte einer ökologischen Reform in Deutschland 1760-1860
}

\section{François Walter}

\section{OpenEdition}

\section{Journals}

Édition électronique

URL : http://journals.openedition.org/ifha/6571

DOI : 10.4000/ifha.6571

ISSN : 2198-8943

Éditeur

IFRA - Institut franco-allemand (sciences historiques et sociales)

Référence électronique

François Walter, « Hölzl, Richard, Umkämpfte Wälder : die Geschichte einer ökologischen Reform in Deutschland 1760-1860 », Revue de l'IFHA [En ligne], Date de recension, mis en ligne le 01 janvier 2011, consulté le 22 septembre 2020. URL : http://journals.openedition.org/ifha/6571 ; DOI : https://doi.org/ 10.4000/ifha.6571

Ce document a été généré automatiquement le 22 septembre 2020.

(C)IFHA 


\title{
Hölzl, Richard, Umkämpfte Wälder : die Geschichte einer ökologischen Reform in Deutschland 1760-1860
}

\author{
François Walter
}

1 En ouverture, l'auteur relate un conflit entre un philosophe promoteur et industriel, Franz von Baader, et les paysans du village de Blachendorf en Bavière, à propos de la mise en valeur d'un espace forestier. Pétri de références rationnelles sur la manière " moderne » de gérer les ressources en bois, le philosophe se heurte à l'opposition des paysans attachés aux antiques droits de parcours. Ils ne sont pas disposés à les céder même si on leur explique les avantages liés à l'installation d'une verrerie qui a besoin de combustible.

2 Cet exemple cadre la problématique de ce gros livre, issu d'une thèse de doctorat soutenue à Göttingen et rédigée dans le cadre privilégié du Graduiertenkolleg " Interdisziplinärer Umweltgeschichte ». C'est donc la réforme forestière qui en constitue l'objet, non sans liens avec des préoccupations écologiques très actuelles, tout en prenant délibérément le parti des acteurs. Il s'agit de savoir comment s'est construit le consensus apparent sur le concept même de réforme forestière. Préalablement, l'auteur évoque, en amont, les nombreuses tensions entre le discours savant qui veut promouvoir l'exploitation rationnelle des forêts et le pragmatisme de populations apparemment hostiles. C'est pourquoi une attention toute particulière est accordée à la masse documentaire qui rend compte des procédures de délits forestiers (Waldfrevel). En effet, tout ce qui s'oppose à la gestion voulue par l'administration est en quelque sorte « criminalisé » et, partant, susceptible de peines de prison ou d'amendes. Comme l'administration du royaume de Bavière va finalement l'emporter, les paysans vont passer pour des rebelles hostiles au progrès et à l'ordre social. Voilà qui accrédite l'image de populations rurales aux attitudes peu écologiques et finalement responsables des crises environnementales, du moins telles que l'on pouvait les percevoir au XIXe siècle. 
Il est particulièrement important de constater comment le discours dominant du siècle des Lumières met en évidence les risques de pénuries des ressources en prétendant développer une forêt durable (nachhaltig) et moderne. En allemand, c'est effectivement dans le contexte forestier du XVIIIe siècle que le concept de « durabilité », devenu bien plus tard durant les années 1980-1990 le paradigme environnementaliste de la postmodernité, a été expérimenté pour la première fois. L'objectif affirmé de l'administration forestière est d'imposer une séparation nette entre les surfaces cultivées et les espaces forestiers, ces derniers entrant dans une logique de mise en valeur selon les lois du marché et donc de privatisation.

4 L'attention prêtée aux acteurs a comme avantage majeur de déconstruire l'argument des tenants de la modernité, à savoir le risque de pénurie énergétique. On trouve confirmation dans ce livre de la thèse qui veut que ce discours a largement été exagéré et instrumentalisé par les élites désireuses de faire passer les réformes. Quant aux acteurs populaires, les paysans, volontiers qualifiés d'archaïques, ils expriment des positions beaucoup plus nuancées en défendant une logique multifonctionnelle de la forêt à l'opposé du productivisme auquel prétendent l'administration et le pouvoir de la bureaucratie étatique. Selon les régions, leur vision de la forêt peut être très diverse et témoigne en tous les cas d'une conception plus complexe du maintien des équilibres écologiques que ne le prétend le discours administratif.

5 L'auteur connaît bien évidemment la très vaste littérature savante imprimée aux XVIIIe-XIXe siècles à propos de l'économie forestière ; il a aussi travaillé dans les archives de Munich, Landshut et Wurtzbourg. C'est grâce à cette enquête dans les sources historiques qu'il peut donner la parole aux acteurs locaux et remettre fondamentalement en question la vision normative dominante en histoire d'économie forestière. Celle-ci, sauf exception, a délibérément choisi le parti des administrations forestières. R.H. reconstruit le rapport à la forêt du point de vue de ses utilisateurs premiers, les communautés locales. De cette manière l'auteur s'inscrit dans ce qu'il considère comme une histoire culturelle de l'environnement, attentive aux formes symboliques et aux variations discursives.

6 Le livre s'articule sur trois moments. Le premier montre comment s'est construit le discours sur la modernité forestière entre 1760 et 1800 . Centré sur deux études de cas dans le royaume de Bavière, le deuxième moment se veut microhistoire des conflits locaux et des délits forestiers entre 1800 et 1860 lorsque l'administration royale essaie d'imposer sa nouvelle vision de la gestion forestière. Le troisième moment, plus bref, se concentre aussi sur la période 1800-1860 pour saisir les effets de la résistance locale sur la poursuite de la politique forestière. L'analyse conduit à un élargissement des problématiques. En effet, peu à peu, l'opinion publique est saisie par un vaste débat sur le paupérisme rural et la question sociale. Dès lors la forêt prend de toutes autres dimensions en assumant de nouvelles fonctions. Le nouveau discours forestier insiste sur son rôle dans l'équilibre des écosystèmes, comme régulateur des grands cycles naturels, et même sur ses fonctions sociales d'espace de loisir. On est donc passé d'un type de discours essentiellement productiviste à un discours qui met au premier plan les fonctions régulatrices de la forêt que les pouvoirs publics doivent dorénavant protéger. La forêt retrouve ainsi la place essentielle qu'elle avait dans la conscience identitaire germanique. Un thème sur lequel ont pu capitaliser aussi bien les auteurs littéraires que les idéologies nationales. Après avoir été désigné comme un prédateur hostile au progrès, le paysan devient le gardien des valeurs traditionnelles de la culture 
allemande. La conclusion de cette remarquable étude est que la perspective choisie d'une histoire environnementale et culturelle révèle la complexité d'un processus que la seule histoire sociale réduisait faussement à un processus linéaire de modernisation.

7 François Walter (université de Genève) 